\title{
Measurement of the Forward-Backward Asymmetry in $\mathrm{Z} / \gamma^{*} \rightarrow \mu \mu$ Events in CMS at $7 \mathrm{TeV}$
}

\author{
Efe Yazgan ${ }^{* \dagger}$ \\ Texas Tech University \\ E-mail: efe.yazgan@cern.ch
}

\begin{abstract}
We present the first measurement of the forward-backward asymmetry $\left(A_{\mathrm{FB}}\right)$ for $\mu^{+} \mu^{-}$pairs produced in proton-proton collisions via an intermediate $Z / \gamma^{*}$ at $\sqrt{s}=7 \mathrm{TeV}$ in the CMS experiment. Our results are based on an integrated luminosity of $198 \mathrm{nb}^{-1}$. The uncorrected forward-backward asymmetry is measured to be $-0.50 \pm 0.40$ in the mass range $40-70 \mathrm{GeV}$, and $0.14 \pm 0.11$ in the mass range 70-110 GeV. The measured values are consistent with POWHEG + PYTHIA + full CMS simulation predictions of -0.03 and 0.01 in these two mass bins.
\end{abstract}

35th International Conference of High Energy Physics - ICHEP2010,

July 22-28, 2010

Paris France

\footnotetext{
*Speaker.

${ }^{\dagger}$ CMS Collaboration
} 


\section{Forward-Backward Asymmetry in $\mathbf{Z} / \gamma^{*} \rightarrow \mu \mu$ Events}

In the Drell-Yan (DY) process $q \bar{q} \rightarrow Z / \gamma^{*} \rightarrow \ell^{+} \ell^{-}$both vector and axial-vector couplings of electroweak bosons to fermions are present. This results in a forward-backward asymmetry in the DY lepton pairs. This asymmetry depends on the di-lepton invariant mass. Deviations from the Standard Model prediction may indicate the existence of a new neutral gauge boson [1, 2, 3]. The differential cross-section for the parton level process is

$$
\frac{d \sigma}{d(\cos \theta)}=A\left(1+\cos ^{2} \theta\right)+B \cos \theta
$$

where $\theta$ is the emission angle of the negative muon relative to the quark momentum in the centerof-mass frame, and $A$ and $B$ depend on the weak isospin and charge of the incoming fermions. The asymmetry parameter $A_{\mathrm{FB}}$, and its statistical error, $\Delta A_{\mathrm{FB}}$ are given by

$$
A_{\mathrm{FB}}=\frac{\sigma_{F}-\sigma_{B}}{\sigma_{F}+\sigma_{B}}=\frac{N_{F}-N_{B}}{N_{F}+N_{B}}=\frac{3 B}{8 A}, \quad \Delta A_{\mathrm{FB}}=\sqrt{\frac{1-A_{F B}^{2}}{N}}
$$

where $\mathrm{N}$ is the total number of events in the corresponding bin.

We use the Collins-Soper frame [4] in order to reduce the effects arising from the non-zero transverse momentum of the incoming quarks. In this frame, $\theta_{C S}^{*}$ is defined to be the angle between the negative muon momentum and the $z^{\prime}$ axis that bisects the angle between $q$ and $\bar{q}$ in the dimuon center of mass frame. $A_{\mathrm{FB}}$ is evaluated using angular distributions of di-muons at different invariant mass bins. Data corresponding to $198 \mathrm{nb}^{-1}$ integrated luminosity do not allow us to do the measurement in many mass bins, therefore, we have done the measurement in two di-muon mass bins of $40<M_{\mu \mu}<70 \mathrm{GeV} / c^{2}$, and $70<M_{\mu \mu}<110 \mathrm{GeV} / c^{2}$. Data sets taken by CMS at $7 \mathrm{TeV}$ collisions selected using an unprescaled high-level single muon trigger that requires the muons to be contained within the pseudo-rapidity $|\eta| \leq 2.1$ with $p_{T}^{\mu}>9 \mathrm{GeV} / \mathrm{c}$. Only runs and lumi-sections that are certified by the CMS Physics Validation and Certification teams are used in the analysis. Monte Carlo DY signal sample is produced by a next-to-leading order generator (POWHEG [5]) with a cut of $20 \mathrm{GeV} / c^{2}$ on the mass of the di-muon system. The PDF set CTEQ66M is used and QED final-state-radiation is incorporated via the parton-shower algorithm PYTHIA [6]. The generated events are reconstructed using the full CMS detector simulation.

We require a pair of opposite charge muons to pass the muon identification and isolation criteria in the offline analysis. Muon isolation is used to distinguish single muons from muons overlapping with jets. For this selection, the scalar sum of $p_{T}$ of all tracks reconstructed in cones of $R=0.3$ around the direction of a muon momentum is required to be less than $3 \mathrm{GeV}$.

Transverse momentum $\left(p_{T}\right)$, pseudo-rapidity $(\eta)$, and mass distribution of the selected dimuon events in data and Monte Carlo are displayed in Figure 1. Figure 2 shows the $\cos \theta_{C S}^{*}$ distribution and the measured and estimated $\mathrm{A}_{\mathrm{FB}}$. This asymmetry has not been corrected for resolution or dilution effects. These effects are important in a precision measurement, but are small compared to the current statistical errors.

The uncorrected forward-backward asymmetry is measured to be $-0.50 \pm 0.43$ in the mass range 40-70 GeV, and $0.14 \pm 0.11$ in the mass range $70-110 \mathrm{GeV}$ using $198 \mathrm{nb}^{-1}$ of data. The measured values are consistent with POWHEG + PYTHIA + full CMS simulation predictions of -0.03 and 0.01 in these two mass bins. 

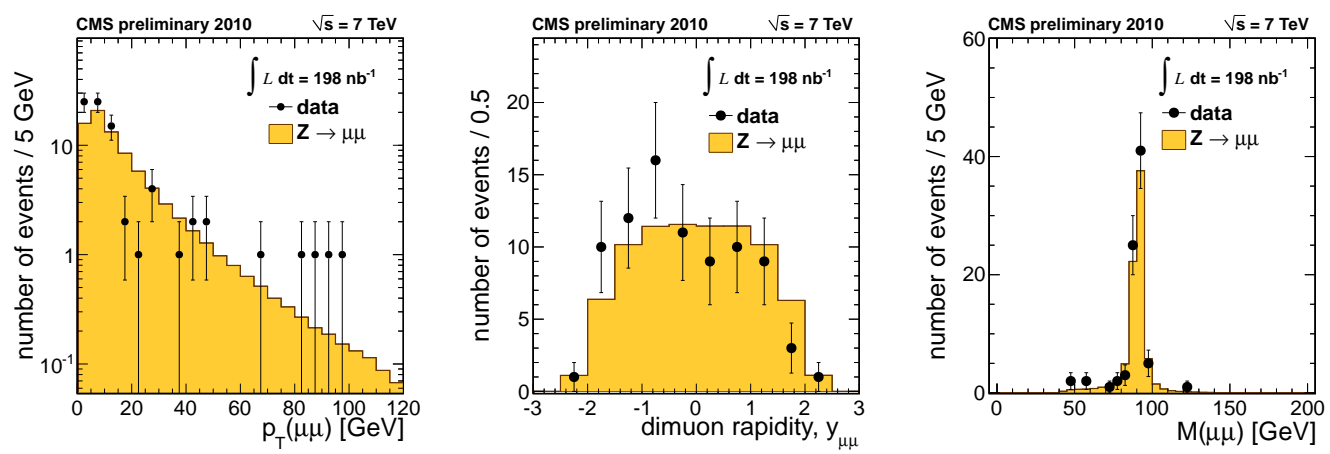

Figure 1: Transverse momentum distribution (left), rapidity distribution of the di-muon events (middle) and mass distribution in MC and data of the di-muon events .
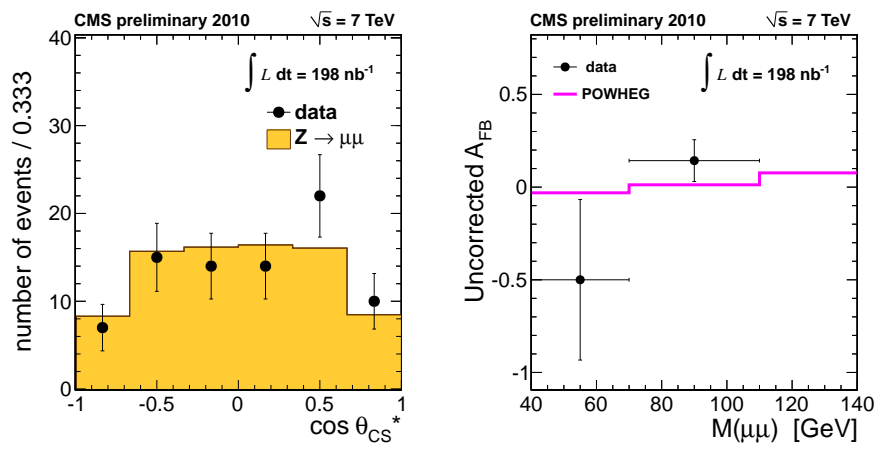

Figure 2: $\cos \theta_{C S}^{*}$ distribution for the di-muon events (left) and measured asymmetry in data with estimated asymmetry in Monte Carlo. Only statistical errors are shown.

\section{References}

[1] London. D., and Rosner, J.L., 'Extra gauge bosons in E6', Phys. Rev. D 34, 1530, 1986.

[2] Rosner, J.L., 'Off-peak lepton asymmetries from new Z's', Phys. Rev. D 35, 2244, 1987.

[3] Cvetic, M., and Godfrey, S., 'Discovery and identification of extra gauge bosons', in: Barklow, T.L (ed.) et al., 'Electroweak Symmetry Breaking and New Physics at the TeV scale' (World Scientific, 1995), 383, [arXiv:hep-ph/9504216].

[4] Collins, J. C. and Soper, D. E., 'Angular distribution of dileptons in high-energy hadron collisions', Phys. Rev. D, 16(7), 2219-2225,1977.

[5] Firixione, S., Nason, P., and Oleari, C., 'Matching NLO QCD Computations with Parton Shower Simulations: the POWHEG Method', JHEP, 11, 070, 2007 [arXiv:0709.2092].

[6] Sjostrand, T.S., Mrenna, S., and Skands, P., 'PYTHIA 6.4 Physics and manual', JHEP 05,026, 2006. 